\section{Consideraciones sobre el programa de detección de diabetes mellitus en población mexicana: el caso del Distrito Federal}

\author{
Analysis of the diabetes mellitus screening \\ program in the Federal District, Mexico
}

\author{
1 Facultad de Medicina \\ Universidad Nacional \\ Autónoma de México, \\ México DF, México. \\ 2 Centro de Estudios y \\ Análisis de la Seguridad \\ Social, México DF, México. \\ Correspondencia \\ M. G. Alvear-Galindo \\ Departamento de Salud \\ Pública, Facultad de \\ Medicina, Universidad \\ Nacional Autónoma de \\ México. \\ Circuito Interior Ciudad \\ Universitaria, Col. Copilco, \\ Delegación Coyoacán, \\ CP 04500, México DF \\ alvear@servidor.unam.mx
}

\begin{abstract}
From the public health perspective, programs to detect type 2 diabetes mellitus are a prime resource for surveillance of the disease. As a screening strategy, the Mexican Ministry of Health implemented the Diabetes Mellitus Action Program (PADM-2), based on two sequential tests: the Risk Factor Questionnaire and capillary blood glucose test. This study explored the Program's capacity as a screening strategy. The study was carried out in the year 2005, with a sample of 1,562 that attended six primary care units under the Health Secretariat of the Federal District. Fasting serum glucose was defined as the gold standard. When assessing the two tests sequentially, sensitivity was $98 \%$, specificity $58.7 \%$, and positive predictive value $16.6 \%$. The positive predictive values indicates that a high proportion of the expenditure still goes to detecting false-positives, thus requiring reconsideration of the Program's criteria to obtain a greater economic and social benefit.
\end{abstract}

Diabetes Mellitus; Health Programs and Plans; Risk Factors
Maria Guadalupe Alvear-Galindo 1

Asa Cristina Laurell 2

\section{Introducción}

La morbilidad y mortalidad por diabetes mellitus tipo 2 se ha incrementado en el mundo y en México 1,2 en los últimos años; su detección muchas veces se realiza en forma tardía y su diagnóstico se efectúa como consecuencia de la presencia de síntomas, ya que en sus etapas iniciales no presenta manifestaciones clínicas. Se estima que cuando se realiza el diagnóstico la enfermedad ha tenido un tiempo de evolución entre 9 y 12 años, y se calcula que no han sido diagnosticados alrededor del $30 \%$ de diabéticos 3 .

En México, en el Programa Nacional de Salud 2007-2012 2 se calcula - usando como parámetro las glucemias en ayunas - que en el país hay más de 5 millones de personas de 20 años y más que padecen diabetes y más de 800 mil de ellas desconocen su condición. Se considera que cerca de $20 \%$ de la población ignora ser diabética, y que el diagnóstico de la enfermedad fue consecuencia de la presencia de diversas complicaciones 3 .

El alto costo económico y social que significa la atención del enfermo diabético para los servicios de salud y para la población en general, así como el deterioro de la calidad de vida del enfermo diabético, y la carga resultante de las complicaciones y mortalidad prematura, es un problema sanitario creciente para la mayoría de los países.

Se han emprendido acciones de promoción a la salud, en particular la prevención de facto- 
res de riesgo asociados a esta enfermedad y a daños cardiovasculares, a través de fomentar el consumo de alimentos sanos y el incremento de la actividad física. Otra medida adoptada para disminuir el gasto en las complicaciones de los enfermos diabéticos ha sido tratar de mejorar su control metabólico, a través del consumo de hipoglucemiantes en la fase considerada como prediabetes 4 .

En México, se calculó que para el año 2005 el gasto en salud destinado a la diabetes mellitus tipo 2 sería de 28.238.104 dólares para el diagnóstico y la consulta médica, 62.940 .675 para medicamentos, 18.870.467 para hospitalización y 52.194.257 para la atención de las complicaciones por diabetes mellitus tipo 2. De costos indirectos se estimó que el gasto sería de 317.631.206 dólares, o sea un total de 162.252 .503 dólares por año 5 .

Por el impacto que tiene en la evolución de la enfermedad la identificación de la población en riesgo en la etapa preclínica, y su control metabólico, se han realizado esfuerzos para identificar la enfermedad en estadios asintomáticos, así como en precisar criterios para el diagnóstico temprano ${ }^{6}$, para lo cual las estrategias de tamizaje han sido primordiales.

Para la identificación de factores de riesgo y su ponderación en la presentación de la diabetes mellitus tipo 2, se han utilizado distintos instrumentos entre los cuales está el Cuestionario de Factores de Riesgo propuesto por la American Diabetes Association (ADA) 7, valorando su sensibilidad, especificidad y el valor predictivo positivo. No obstante, no existe un consenso respecto a su utilidad 8,9,10,11.

En la mayoría de los estudios se ha visto que los distintos instrumentos de detección tienen un valor predictivo positivo (VPP) bajo. En general, los resultados encontrados reportan que la sensibilidad se sitúa en un rango que va del $46 \%$ al $84 \%$, una especificidad entre $50 \%$ y $96 \%$ y un VPP entre $5 \%$ y 13,7\% 10,11,12. Entre los factores de riesgo, identificados como marcadores de riesgo para la presentación de la diabetes mellitus tipo 2, se encuentran el índice de masa corporal (IMC) y la circunferencia de cintura 13 .

Asimismo, se ha visto una asociación positiva entre la edad y la presentación de diabetes mellitus tipo 2; encontrando que en la actualidad ésta se presenta en población cada vez más joven. Igualmente, los criterios en cuanto a los exámenes de laboratorio utilizados y los niveles de glucosa considerados anormales se han modificado ${ }^{14}$, con lo cual han cambiado los criterios para diferenciar enfermos de sanos.

Con el objeto de reconocer en la población a individuos asintomáticos con alta probabilidad de ser diabéticos por la presencia de factores de riesgo, se han establecido diversos programas de tamizaje en distintos países 15,16 e incluso estrategias a nivel nacional como es el caso de Brasil 17.

Dada la alta prevalencia de la diabetes mellitus tipo 2 y el incremento de la frecuencia de sus factores de riesgo en México, como parte del Programa Nacional de Salud 2001-2006 18, la Secretaría de Salud instituyó el Programa de Acción Diabetes Mellitus (PADM-2) 19, donde se considera prioritaria la prevención primaria de la diabetes mellitus tipo 2 y la detección temprana de enfermos diabéticos.

Este programa consiste fundamentalmente en la realización de un tamizaje con dos pruebas secuenciales. La primara prueba consiste en la aplicación de un cuestionario de factores de riesgo y la segunda prueba en la valoración de los niveles de la glucosa capilar y, finalmente, a los individuos identificados como sospechosos efectuarles la confirmación diagnóstica a través de la medición de los niveles de glucosa sérica en ayuno. Por la importancia económica y social que significa este esfuerzo, el objetivo de este trabajo es explorar la capacidad del PADM-2 como estrategia de tamizaje.

\section{Métodos}

\section{Selección de la población}

Este trabajo forma parte de un estudio más grande en donde se probó un sistema de vigilancia epidemiológica para diabetes mellitus tipo 2 en 19 unidades de atención primaria de la Secretaría de Salud del Distrito Federal, con 7.857 sujetos que acudieron a esta institución. En este trabajo participaron 1.562 sujetos que asistieron a seis de las unidades de atención primaria que disponían de laboratorio de análisis clínicos para la determinación de glucosa sérica.

El total de la población fue cubierta por cuotas con las personas que asistieron a las unidades de atención primaria participantes, ya sea como acompañantes o a solicitar atención médica por otro tipo de padecimiento diferente a la diabetes mellitus tipo 2 o por alguna de sus complicaciones. El periodo de captación de voluntarios fue del 10 de marzo al 10 de junio del año 2005.

Todos los sujetos que participaron en este trabajo desconocían ser enfermos diabéticos tipo 2, con edad de 20 años y más, que presentaron alguno de los factores de riesgo considerados en el cuestionario o, que independientemente de de los factores de riesgo, manifestaron tener la sintomatología de poliuria, polidipsia y polifagia 
$\mathrm{y}$, estuvieron dispuestos a contestar las preguntas $\mathrm{y}$, someterse a las determinaciones de glucosa capilar y glucosa sérica en ayuno.

\section{Descripción de la aplicación del PADM-2}

Al PADM-2 ingresan todos los sujetos de 45 años y más, y personas de 20 a 44 años que presenten alguno de los factores de riesgo incluidos en el cuestionario, así como aquellos sujetos que manifiesten tener poliuria, polidipsia y polifagia.

Como ya se mencionó, el esquema de tamizaje consiste en la aplicación de dos pruebas dispuestas de manera seriada. En la primera, a través del cuestionario se explora la presencia de factores de riesgo para diabetes mellitus tipo 2 y la sintomatología de poliuria, polidipsia y polifagia y, en la segunda prueba, se miden los niveles de glucosa capilar, para finalmente -en aquellos sujetos que se consideran sospechosos de presentar la enfermedad - proceder a la confirmación diagnóstica a través de la medición de los niveles de glucosa sérica en ayuno (Figura 1).

Se consideran variables incluidas en el cuestionario propuesto por la ADA 7 como son edad, sexo, antecedentes familiares, para las mujeres haber tenido hijos de cuatro kilos o más, características de la actividad física y características antropométricas.

Figura 1

Programa de tamizaje utilizado en los Servicios de Salud. Secretaría de Salud del Distrito Federal, México, 2005 *.

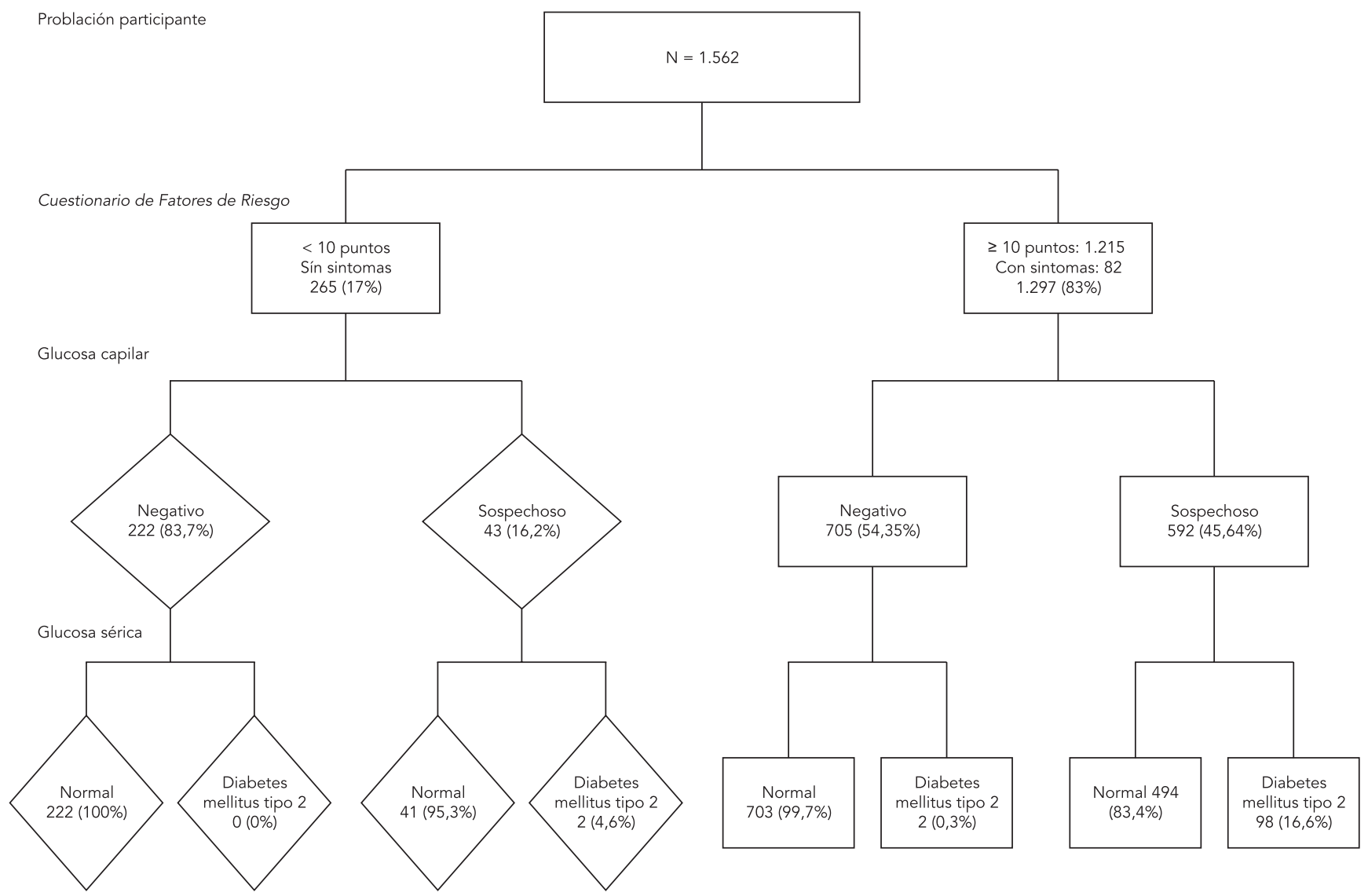

Nota: prevalencia total: 6.53\%; prevalencia de síntomas: 5,2\%; prevalencia de población en riesgo: 7,7\%; prevalencia de población sin riesgo: 0,75\%; prevalencia de población con $\geq 10$ puntos: $7,7 \%$.

* En el Programa de Acción Diabetes Mellitus de la Secretaría de Salud Federal, solamente sigue con la segunda prueba diagnóstica la población considerada con mayor riesgo de presentar diabetes mellitus tipo 2. 
Para ponderar la importancia de estas variables, se utiliza una escala de riesgo con valores que van del 0 al 10; la suma de los valores de cada una de las variables del cuestionario oscila entre 1 y 37. Con este instrumento también se explora la presencia de la sintomatología mencionada (Tabla 1). En este trabajo, el cuestionario fue aplicado por el personal de enfermería de las unidades de atención primaria.

En el PADM-2 19 se considera como población en riesgo a todos los individuos de 45 años y más, y a los mayores de 20 años que en el cuestionario alcanzan un puntaje de $\geq 10$ o a sujetos que, independientemente del puntaje de riesgo obtenido, manifiesten tener sintomatología positiva. Se califica como población sin riesgo a los sujetos que obtienen un puntaje de $<10$ y no manifiestan presentar sintomatología.

Como lo señala el PADM-2 19, a las personas que alcanzan un puntaje $\geq 10$ se les considera como sospechosas de ser enfermos diabéticos y continúan con la siguiente prueba del tamizaje, que consiste en la medición de la glucemia capi- lar. Si la persona refiere presentar sintomatología, independientemente de la edad, del puntaje de riesgo obtenido, y de los niveles de la glucosa capilar, se clasifica como sospechoso y se le realiza la prueba de confirmación diagnóstica.

Si el individuo no alcanza un puntaje de riesgo, es considerado como normal y en el programa también se especifica que - según sus características - deberá regresar para una nueva valoración. Así, si son mayores de 45 años, se les sugiere volver al año siguiente y, si es mayor de 20 y menor de 45 años, pero presenta algún factor de riesgo, la recomendación es que regrese a los tres años.

En este trabajo, para identificar el status de la enfermedad en la población participante y precisar el porcentaje de diabéticos, así como para determinar la sensibilidad, la especificidad y el VPP de la estrategia de detección de diabetes mellitus tipo 2, se aplicó el esquema de tamizaje completo a toda la población participante, es decir, la cumplimentación del cuestionario de factores de riesgo y las mediciones de la glucemia capilar y

Tabla 1

Características de la población por variables del Cuestionario de Factores de Riesgo 7 según categoría de riesgo. Secretaría de Salud del Distrito Federal, México, 2005.

\begin{tabular}{|c|c|c|c|c|c|}
\hline Factor de riesgo & $\begin{array}{c}\text { Escala de } \\
\text { riesgo }\end{array}$ & $<10$ & $\geq 10$ & $\begin{array}{l}\text { Sin factor } \\
\text { de riesgo }\end{array}$ & Total \\
\hline \multicolumn{6}{|l|}{ Sexo } \\
\hline Masculino & 0 & 105 & 252 & - & 357 \\
\hline Femenino & 0 & 242 & 963 & - & 1.205 \\
\hline \multicolumn{6}{|l|}{ Antecedentes familiares } \\
\hline Hermano(s)con diabetes & 1 & 40 & 243 & 1,279 & 1.562 \\
\hline Padres con diabetes & 1 & 109 & 451 & 1,002 & 1.562 \\
\hline Mujer con hijos de cuatro kg y más * & 1 & 11 & 113 & 1,081 & 1.205 \\
\hline Poco o nada de ejercicio ** & 5 & 127 & 603 & 716 & 1.446 \\
\hline \multicolumn{6}{|l|}{ Edad (años) } \\
\hline $20-44$ & 0 & 298 & 708 & 1,006 & 1.006 \\
\hline $45-64$ & 5 & 39 & 401 & 0 & 440 \\
\hline$\geq 65$ & 9 & 10 & 106 & 0 & 116 \\
\hline \multicolumn{6}{|l|}{ Clasificación de índice de masa corporal } \\
\hline Normal & 0 & 297 & 1 & 399 & 399 \\
\hline Sobrepeso & 5 & 50 & 193 & 0 & 243 \\
\hline Obesidad & 10 & 0 & 920 & 0 & 920 \\
\hline \multicolumn{6}{|l|}{ Circunferencia cintura } \\
\hline Masculino $>95 \mathrm{~cm} /$ Femenino $\geq 85 \mathrm{~cm}$ & 10 & 0 & 1.023 & 539 & 1.562 \\
\hline \multicolumn{6}{|l|}{ Presencia de síntomas } \\
\hline Sí & - & 82 & 385 & - & 467 \\
\hline No & - & 265 & 830 & - & 1.095 \\
\hline
\end{tabular}

* Se incluyen solamente mujeres;

** No se incluyeron sujetos mayores de 65 años (116 sujetos) como lo establece el Cuestionario de Factores de Riesgo. 
sérica, independientemente del valor obtenido en la escala de riesgo y/o la presencia de síntomas, así como de los niveles de glucosa capilar alcanzados.

\section{Mediciones en laboratorio}

Para medir la glucemia capilar, se utilizaron glucómetros de la marca Accu-Chek modelo Sensor, con lancetas y tiras reactivas para dicho equipo, todos del Laboratorio Roche S. A. (Suiza). Los grados de funcionalidad del glucómetro y tiras reactivas fueron sujetos a un protocolo externo de certificación avalado por el Instituto Politécnico Nacional 20. La clasificación de la glucemia capilar en ayuno o casual, se determinó de acuerdo con los criterios establecidos por la ADA 7, que son los mismos que se utilizan en el PADM-2 19, donde se clasifica al sujeto como positivo o sospechoso, cuando la glucosa capilar en ayuno es $\mathrm{de} \geq 100 \mathrm{mg} / \mathrm{dL}$ o la glucosa capilar casual es de $\geq$ $140 \mathrm{mg} / \mathrm{dL}$, en caso de ser inferior a estos puntos de corte el resultado de la prueba se considera negativo.

En el PADM-2 19, el resultado de la glucosa sérica en ayuno, también se clasifica de acuerdo a uno de los criterios de la ADA 7, que establece como resultado negativo o normal si la glucosa sérica en ayuno es de $<126 \mathrm{mg} / \mathrm{dL}$; y positivo o confirmado de si la glucosa sérica en ayuno alcanza valores de $\geq 126 \mathrm{mg} / \mathrm{dL}$. En este trabajo, se utilizó como valor de referencia el nivel de glucosa sérica en ayuno, que fue medido en los laboratorios de cada una de las unidades de atención primaria de la Secretaría de Salud del Distrito Federal, con equipos microlab 100 (Merk, Alemania) dispuestos en cada sede participante. Se utilizaron los mismos reactivos y lotes en todos los equipos.

\section{Análisis estadístico}

Para conocer el comportamiento de los factores de riesgo, la presencia de síntomas y los niveles de glucosa capilar se clasificó a la población en base al puntaje obtenido y, para saber cual era la correspondencia entre los criterios de tamizaje con el criterio de confirmación diagnóstica, se compararon estos con los niveles de la glucosa sérica en ayuno.

Se definió la sensibilidad como la proporción o porcentaje de sujetos enfermos que fueron identificados como positivos mediante la prueba diagnóstica; la especificidad como la proporción o porcentaje de sujetos sin la enfermedad que fueron identificados como negativos mediante la prueba diagnóstica. El VPP se determinó como la proporción de sujetos con prueba de diagnóstico positiva y que fueron diagnosticados con la enfermedad; el valor predictivo negativo (VPN) como la proporción de sujetos con prueba de diagnóstico negativa y que no tenían la enfermedad.

Se calculó la sensibilidad, la especificidad y el VPP y VPN para la población que se clasificó como de riesgo por obtener un puntaje de riesgo de $\geq 10$ y/o la presencia de síntomas; para la población que se catalogó como de riesgo sólo por la presencia de síntomas; y para la población que se consideró de riesgo exclusivamente por alcanzar un puntaje de $\geq 10 \mathrm{y}$, finalmente para los sujetos que presentaron glucemia capilar positiva.

Los valores predictivos se calcularon con la prevalencia de $8.5 \%$ para diabetes mellitus tipo 2 que es la estimada por la Encuesta Nacional de Salud 2000 (ENSA 2000) 21. Para conocer el comportamiento de los VPP y VPN se calcularon estos con distintas prevalencias, con los valores de sensibilidad y especificidad encontrados en este trabajo. El programa usado fue el Epidat versión 3.1 (Xunta de Galicia, España; http://dxsp.sergas. es/default.asp), utilizando el teorema de Bayes.

Mediante una curva ROC (receiver operating characteristics) que caracteriza la relación entre la tasa de los verdaderos positivos (sensibilidad) y la tasa de los verdaderos negativos (1-especificidad) se estimó el área bajo la curva para cada una de las poblaciones.

El área bajo la curva ROC se definió como el porcentaje de sujetos correctamente clasificados mediante el tamizaje como diabéticos (con la enfermedad) o como no diabéticos (sin la enfermedad). Los cálculos se realizaron con un intervalo de confianza al 95\% (IC95\%). Para el análisis se utilizó el paquete estadístico Stata versión 6.0 (Stata Corp., College Station, Estados Unidos).

\section{Resultados}

Descripción de la población de estudio y selección de la población por las pruebas en serie

Como se muestra en la Figura 1, de los 1.562 participantes que respondieron el cuestionario, el 83\% ingresó PADM-2 19 por alcanzar un puntaje de $\geq 10$ o por manifestar sintomatología, de éstos el 45,6\% obtuvo niveles de glucosa capilar que los definían como sospechosos y, de éstos el 16\% se confirmaron como enfermos diabéticos. En los sujetos que habían obtenido valores negativos en la glucemia capilar, dos clasificaron como enfermos diabéticos por los valores obtenidos en la medición de la glucosa sérica.

De los 265 sujetos que calificaron sin riesgo con el cuestionario, el $83 \%$ obtuvo niveles de glu- 
cosa capilar y glucosa sérica con valores negativos y, al 4,6\% de los sospechosos se les confirmó como enfermos diabéticos con la medición de la glucosa sérica.

La prevalencia identificada en el total de la población participante por los niveles de la glucosa sérica en ayuno fue de $6,53 \%$ con un IC95\%: 5.35-7.87, mientras que la prevalencia en el grupo de mayor riesgo fue de $7,7 \%$, con dos casos $(0,8 \%)$ con resultados positivos en la población de menor riesgo.

\section{Comportamiento de los factores de riesgo en la población}

Como se muestra en la Tabla 1, del total de los participantes, el $77,1 \%$ correspondió a la población femenina. El 79,9\% de las mujeres y el 70,6\% de los hombres obtuvieron un puntaje de riesgo. La edad osciló entre los 20 y los 86 años, el $64 \%$ del total tenía una edad entre 20 y 44 años, el promedio de edad para mujeres fue de 41 años y para hombres de 42 años.

Alcanzar un puntaje de riesgo y tener hermanos con diabetes se presentó en el 15,5\% de los participantes, tener padres con diabetes en el $28,8 \%$, y de las mujeres que se consideraron como de riesgo el 9,3\% tuvo hijos de más de cuatro kilos.

Si bien, la actividad física es sumamente difícil de valorar, esta se estimó con la pregunta: “ ¿Usted realiza poco o nada de ejercicio?” lo cual hace más imprecisa su valoración; el 49,5\% contestó que realizaba poco o nada de ejercicio.

En cuanto a la forma de la distribución del puntaje por edad, el $64 \%$ del total de la población se situó en el grupo de 20 a 44 años de edad, el cual dentro de la escala de riesgo tiene un valor de 0 , sin embargo, el 70,4\% de este grupo de edad ingresó en el programa de tamizaje por puntaje de riesgo de $\geq 10$. El $95 \%$ de la población de 45 y más alcanzó un puntaje de riesgo, así independientemente de la edad como factor de riesgo, en los tres grupos etáreos se presentaron otras características que los clasificaron como de riesgo.

De los 1.562 sujetos, el 58,9\% ingresó el PADM-2 19 por presentar obesidad y el $15,6 \%$ por sobrepeso. De éstos últimos, el 79,4\% alcanzó un puntaje de $\geq 10$. El perímetro de cintura -considerado de riesgo - se encontró en 1.023 sujetos, por lo que se puede suponer que el $78 \%$ de la población ingresada en el programa fue por presentar este factor, y únicamente 274 (13,4\%) por algún otro factor o por la presencia de más de uno de éstos.

\section{Valores de la glucosa capilar}

De los 1.562 sujetos que participaron en este trabajo, $635(40,7 \%)$ obtuvo niveles de glucosa capilar considerados de sospechoso a positivo y el resto niveles de negativo a normal.

De los 1.297 individuos calificados como de riesgo a través del cuestionario, 705 (54\%) obtuvieron valores normales de glucosa capilar, 592 obtuvieron valores de sospechoso a positivo. Así, el $45,6 \%$ de los sujetos que ingresaron y pasaron por las dos fases del programa de tamizaje habrían continuado con la confirmación diagnóstica sólo por sus niveles de glucosa capilar, independientemente del resultado del cuestionario de factores de riesgo.

\section{Criterios del tamizaje y confirmación diagnóstica}

Cuando se valoraron los niveles de glucosa sérica, se encontró que del total de los sujetos identificados como de riesgo por el cuestionario, el 92,3\% presentó valores de glucosa sérica en ayuno de $<126 \mathrm{mg} / \mathrm{dL}$ y el $7,7 \%$ niveles de glucosa sérica en ayuno de $\geq 126 \mathrm{mg} / \mathrm{dL}$. El 2,3\% de los sujetos, considerados por el cuestionario como de bajo riesgo, registró cifras $\geq 126 \mathrm{mg} / \mathrm{dL}$ de glucosa sérica en ayuno y dos sujetos resultaron falsos negativos.

$\mathrm{Al}$ considerar únicamente la sintomatología como criterio de riesgo, el 11,3\% obtuvo valores de glucosa sérica anormales. Al relacionar los niveles de la glucemia capilar con los niveles de glucosa sérica se observó que, de los 535 que resultaron positivos en la primera medición, el $15,7 \%$ obtuvo valores de glucosa sérica anormales. Al combinar puntaje de riesgo y síntomas el resultado fue semejante al obtenido sólo con el cuestionario de factores de riesgo (Tabla 2).

De los sujetos que resultaron positivos en las dos pruebas del tamizaje, el 16,6\% tuvieron niveles de glucosa sérica que los clasificó como enfermos diabéticos.

\section{Sensibilidad, especificidad y otros indicadores de las pruebas del tamizaje}

Al valorar la eficiencia del programa de detección de diabetes mellitus tipo 2, con únicamente la escala de riesgo se alcanzó una sensibilidad de $92,1 \%$ y una especificidad de $23,2 \%$; al combinar la escala de riesgo con la presencia de síntomas, la sensibilidad aumentó a 98\% y, la especificidad bajó a 18\%. En ambos casos, el VPP fue de 7,7\% (Tabla 3).

Para la población que únicamente manifestó sintomatología, la sensibilidad fue de 51,9\%, la 


\section{Tabla 2}

Criterios de programa de detección y estándar de oro ( $N$ = 1.562). Secretaría de Salud del Distrito Federal, México, 2005.

\begin{tabular}{|c|c|c|c|c|c|}
\hline \multirow[t]{3}{*}{ Criterio de tamizaje } & \multirow[t]{3}{*}{ Clasificación del riesgo } & \multicolumn{4}{|c|}{ Glucosa sérica en ayuno $(\mathrm{mg} / \mathrm{dL})$} \\
\hline & & \multicolumn{2}{|c|}{$<126$} & \multicolumn{2}{|c|}{$\geq 126$} \\
\hline & & $\mathrm{n}$ & $\%$ & $\mathrm{n}$ & $\%$ \\
\hline \multicolumn{6}{|l|}{ Puntaje de riesgo } \\
\hline & Bajo (< 10 puntos) & 339 & 97,7 & 8 & 2,3 \\
\hline & Alto ( $\geq 10$ puntos) & 1.121 & 92,3 & 94 & 7,7 \\
\hline \multicolumn{6}{|l|}{ Presencia de síntomas } \\
\hline & No & 1.046 & 95,5 & 49 & 4,5 \\
\hline & Sí & 414 & 88,7 & 53 & 11,3 \\
\hline \multicolumn{6}{|l|}{ Glucemia capilar } \\
\hline & Negativa & 925 & 99,8 & 2 & 0,2 \\
\hline & Positiva & 535 & 84,3 & 100 & 15,7 \\
\hline \multicolumn{6}{|c|}{ Puntaje de riesgo y presencia de síntomas } \\
\hline & No & 263 & 99,2 & 2 & 0,8 \\
\hline & Sí & 1.197 & 92,3 & 100 & 7,7 \\
\hline \multicolumn{6}{|c|}{$\begin{array}{l}\text { Puntaje de riesgo, presencia de síntomas y } \\
\text { glucemia capilar* }\end{array}$} \\
\hline & Negativo & 703 & 99,7 & 2 & 0,3 \\
\hline & Positivo & 494 & 83,4 & 98 & 16,6 \\
\hline
\end{tabular}

* Sigue el proceso de prueba en serie establecido por el Programa de Acción Diabetes Mellitus (PADM-2) 19.

Tabla 3

Sensibilidad, especificidad, valor predictivo positivo (VPP), valor predictivo negativo (VPN) y área bajo la curva. Secretaría de Salud del Distrito Federal, México, 2005.

\begin{tabular}{|c|c|c|c|c|c|c|}
\hline \multirow[t]{2}{*}{ Prueba de tamizaje } & \multirow{2}{*}{$\begin{array}{c}\text { Sensibilidad } \\
\%\end{array}$} & \multirow{2}{*}{$\begin{array}{c}\text { Especificidad } \\
\%\end{array}$} & \multirow{2}{*}{$\begin{array}{l}\text { VPP } \\
\%\end{array}$} & \multirow{2}{*}{$\begin{array}{c}\text { VPN } \\
\%\end{array}$} & \multicolumn{2}{|c|}{ Área bajo la curva } \\
\hline & & & & & $\%$ & IC95\% \\
\hline Puntaje de riesgo & 92,1 & 23,2 & 7,7 & 97,7 & 57,7 & $54,8-60,5$ \\
\hline Presencia de síntomas & 51,9 & 71,6 & 11,3 & 95,5 & 61,8 & $56,8-66,8$ \\
\hline Glucemia capilar & 98,0 & 63,4 & 15,8 & 99,8 & 80,7 & $78,8-82,5$ \\
\hline Puntaje de riesgo y presencia de síntomas & 98,0 & 18,0 & 7,7 & 99,3 & 58,0 & $56,4-59,7$ \\
\hline Puntaje de riesgo, presencia de síntomas y glucemia capilar & 98,0 & 58,7 & 16,6 & 99,7 & 78,4 & $76,4-80,3$ \\
\hline
\end{tabular}

* Sigue el proceso de prueba en serie establecido por el Programa de Acción Diabetes Mellitus (PADM-2) 19.

especificidad de 71,6\%, y el VPP fue de $11,3 \%$. Al valorar las dos pruebas en serie del esquema de tamizaje, con los criterios señalados en el programa de detección de diabetes mellitus tipo 2 se obtuvo una sensibilidad de $98 \%$, una especificidad de 58,7\%, y un VPP de 16,6\%.

Cuando se calcularon los VPP y VPN con distintas prevalencias para diabetes mellitus tipo 2 para la población estudiada, por ejemplo, para una prevalencia del $10 \%$ el VPP fue de $23,83 \%$ y el VPN fue de $99,61 \%$, con una prevalencia de $12 \%$ el VPP fue de $24,48 \%$ y el VPN de $99,55 \%$, y con la prevalencia del $14 \%$ elVPP fue de $33,46 \%$ y elVPN de 99,48\% (Figura 2).

La población clasificada sólo por síntomas registró un área bajo la curva ROC de 61,8\%. El 
Figura 2

Valores predictivos positivos (VPP) y negativos (VPN) según prevalencias estimadas en la detección de diabetes mellitus tipo 2. Secretaría de Salud del Distrito Federal, México.

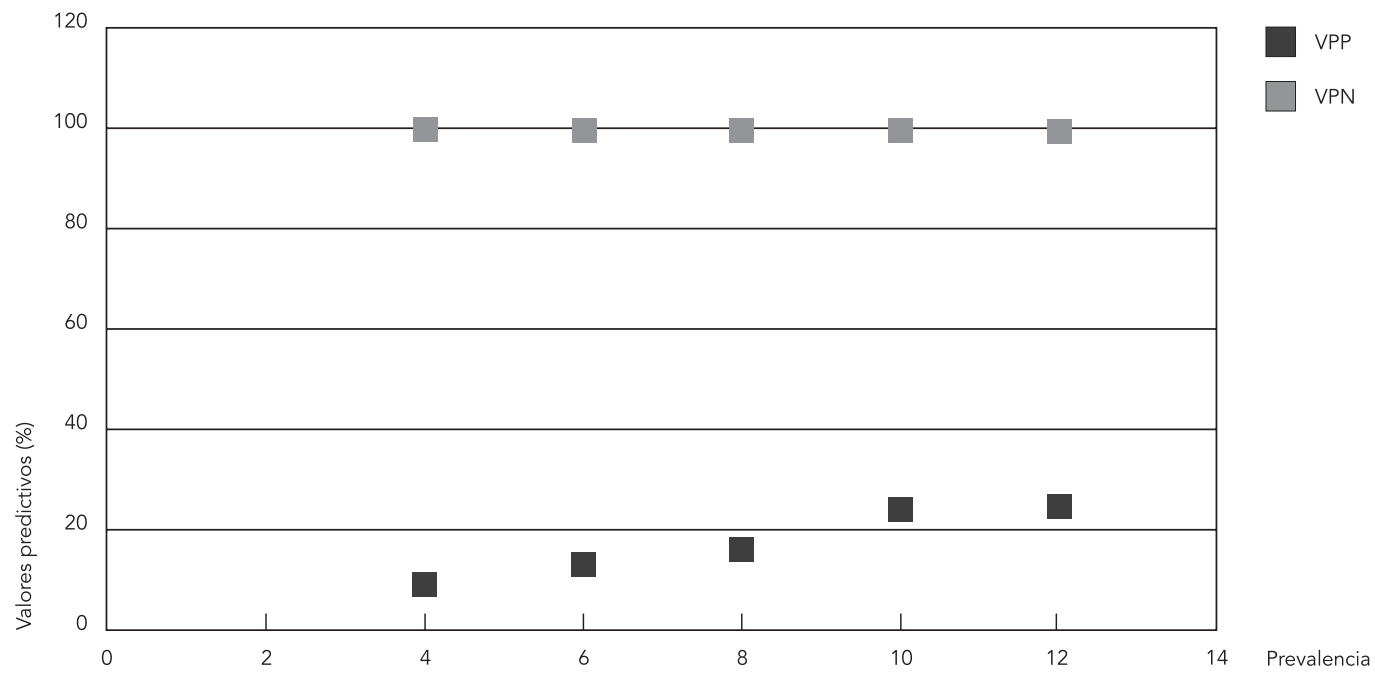

valor encontrado con la curva ROC para el esquema de tamizaje completo fue del $78,4 \%$, y el valor más alto fue para la glucemia capilar con el $80,7 \%$ del área bajo la curva (Tabla 3).

\section{Discusión}

Desde el ámbito de la salud pública, los programas de detección para diabetes mellitus tipo 2 son un recurso para su vigilancia, ya que, al identificar características y grupos de riesgo, así como al estimar la capacidad de los esquemas de tamizaje a través de valorar su sensibilidad, su especificidad y el VPP, es factible disminuir los costos directos que incluyen atención al paciente, gastos de laboratorio, hospitalización y medicamentos, así como los costos sociales 22.

Con el fin de identificar a población en riesgo para diabetes mellitus tipo 2, la ADA 7 propuso un cuestionario, a partir del cual se han planteado diferentes instrumentos con criterios y escalas de medición distintas, con el fin reconocer el comportamiento de las características de la población y ponderar y definir en qué punto estas características pueden ser consideradas como riesgos para la diabetes mellitus tipo 2 .

El PADM-2 19 propuesto por la Secretaría de Salud Nacional con el cuestionario de factores de riesgo utilizado 7 , ofrece la posibilidad de selec- cionar a población con características de riesgo, así como a sujetos que manifiestan presencia de síntomas de la enfermedad. Así, con esta estrategia se realiza un tamizaje al identificar a sujetos asintomáticos a partir de su grado de riesgo, y una prueba diagnóstica al considerar individuos que manifiestan sintomatología.

En algunos trabajos se ha considerado que las mediciones antropométricas son difíciles de valorar puesto que estas varían con la edad, el sexo y el grupo étnico entre otros y, se ha confirmado la asociación entre IMC, circunferencia de cintura y relación cintura/cadera con la presencia de diabetes mellitus tipo 2. Asimismo, se ha visto que el riesgo de ser diabético se incrementa con el aumento del IMC y, en particular, se ha encontrado una fuerte asociación entre la obesidad central y el aumento de riesgo de diabetes mellitus tipo 2 12,22.

Entre los factores de riesgo explorados en este trabajo, los de mayor peso fueron la obesidad y la circunferencia de cintura, lo cual coincide con lo reportado en otros estudios $23,24,25$. Se considera que el incremento de la frecuencia del sobrepeso y la obesidad son responsables del incremento de la prevalencia de diabetes mellitus tipo 2, por lo que es fundamental señalar que el sobrepeso y la obesidad se presentó en tres cuartas de la población en riesgo identificada en este trabajo. 
Si bien, del total de la población, el $83 \%$ tuvo una circunferencia de cintura de riesgo e ingresó automáticamente al tamizaje - lo cual hace muy sensible al cuestionario - afecta la especificidad, que es favorecida por la facilidad de alcanzar el puntaje de riesgo. En otros trabajos, con el objeto de revalorar su peso como factor de riesgo de diabetes mellitus tipo 2 , se ha sugerido evaluar distintos puntos de corte para la circunferencia de cintura, por lo que se considera fundamental reconsiderar la forma en que se ponderan las mediciones antropométricas.

Se reconoce a la edad como factor de riesgo para presentar diabetes mellitus tipo 2, y se observa un aumento directamente proporcional entre ésta y la presentación de la enfermedad. En este trabajo, el $70,4 \%$ de la población de 20 a 44 años de edad calificó con un puntaje de $\geq 10 \mathrm{y}$, el promedio de edad fue de 41 años, con lo cual se puede suponer que la edad como se agrupa y califica en el cuestionario, no es suficiente para definir a sujetos en riesgo por este factor.

Ya en otros trabajos se ha reconocido como población en riesgo para diabetes mellitus tipo 2 a niños y adolescentes, utilizando criterios específicos para este grupo de edad como es el estimar el IMC por edad y sexo a partir de valores percentilares. La ADA sugiere tamizar con mediciones de glucosa plasmática a jóvenes con perfil de riesgo para diabetes mellitus tipo 26 .

La ENSA 200021 y la Encuesta Urbana de Alimentación 200227 reportan que el 59\% de la población se encuentra entre los 20 y 40 años de edad. Por lo que probar otros puntos de corte para la edad podría ayudar a reducir a los falsos positivos. Así, se podría considerar la posibilidad de disminuir la edad de 45 años como criterio de riesgo, lo cual podría aumentar la especificidad de este factor.

Por la importancia que tienen la actividad física y la conducta alimentaria, como factores de riesgo de diabetes mellitus tipo 2, se siguen explorando distintas formas de obtener información de manera rutinaria sobre estos factores. Así, en algunos cuestionarios de riesgo para diabetes mellitus tipo 2, se han incorporado preguntas que posibilitan valorar en el primer nivel de atención, el comportamiento de estas variables en la población 28.

Aunque no se hizo una valoración en cuanto al constructo de las preguntas del cuestionario, se considera que la forma en que se estructuran algunas de ellas como, por ejemplo, para explorar actividad física o la sintomatología no favorece su estimación, por lo que se considera necesario incluir otro tipo de preguntas que ayuden a reconocer el comportamiento de los factores de riesgo señalados $\mathrm{y}$ - por la importancia que tiene el sobrepeso y obesidad en esta población - incluir algunas preguntas que ayuden a identificar la conducta alimentaria.

En otros estudios se están considerando otro tipo de variables en la construcción de escalas de riesgo 28,29. Asimismo, la ENSA 200021 ya reporta que la presencia de diabetes mellitus tipo 2 se presenta con mayor frecuencia en los grupos con menor escolaridad. Como una primera tarea, se podrían revisar los criterios y escalas de medición de los factores de riesgo incluidos en el cuestionario de factores de riesgo, así como, incorporar variables que, en otros trabajos se han encontrado como riesgos de diabetes mellitus tipo 2 como es la escolaridad y el nivel socioeconómico 30.

Bajo el criterio de sintomatología, el cuestionario detecta a la mitad de los sujetos enfermos (51,9\%), cuando en realidad se esperaba que identificara a los enfermos como tales, porque la presencia de sintomatología es evidencia cierta de que la diabetes mellitus tipo 2 lleva en evolución entre 4 y 7 años. La sensibilidad encontrada para la detección temprana es de un $98 \%$ y dos sujetos, que a través de este instrumento se diagnosticaron como sanos, presentaron valores de glucosa sérica en ayuno por arriba de 126mg/ dL. Para optimizar el instrumento como prueba diagnóstica, se podrían integrar otros síntomas de hiperglicemia como son la disminución de peso y la visión borrosa.

En otros cuestionarios donde se exploran factores de riesgo para diabetes mellitus tipo 2 , similares a los incluidos en este cuestionario, se ha encontrado una sensibilidad de $79 \%$, una especificidad de $65 \%$, un VPP de $10 \%$ y un área bajo la curva de 0,78 y, variando las escalas de riesgo, se han encontrado valores de sensibilidad que van de $66,7 \%$ a $79,3 \%$ de especificidad de $68,7 \%$ a $78 \%$ y, VPP entre $7,7 \%$ a $12 \% 22,23$. En este trabajo, para el cuestionario de factores de riego los valores de la sensibilidad, la especificidad y el VPP encontrados están dentro de los valores mencionados.

En cuanto a los resultados sobre los niveles de glucosa capilar, se encontró que coinciden con lo reportado en un tamizaje realizado en Brasil, donde el 15,7\% de las mediciones de glucosa capilar fueron positivas con un rango en la tasa de positividad de $11,3 \%$ a $25,3 \% 17$. Con estos resultados se puede asumir que la primera prueba tiene una mayor sensibilidad y la segunda una mayor especificidad.

Al contrastar los valores predictivos con diferentes prevalencias, se encontró que al aumentar los valores de éstas, los VPP aumentaban y los VPN disminuían, lo cual coincide en lo reportado en otro trabajo 31; sin embargo, al comparar los resultados obtenidos con datos referidos por 
la Organización Mundial de la Salud (OMS) 32 sobre un estudio de detección de diabetes mellitus tipo 2 con pruebas secuenciales en población mexicana, se encontró que los valores predictivos eran estadísiticamente diferentes, lo cual puede deberse a limitaciones propias de este trabajo, a diferencias en los valores de la sensibilidad y la especificidad de las pruebas utilizadas, así como al tamaño muestral, ya que el número de participantes en este estudio es significativamente menor que el de los trabajos señalados en el documento de la OMS.

Entre las limitaciones que puede tener este trabajo es que éste se realizó de manera local, es decir, solamente en la Ciudad de México; así como en la forma en que se seleccionó la población participante, ya que ingresó en este estudio solamente aquella que acudió a los servicios de salud de la Secretaría de Salud del Distrito Federal, con lo cual probablemente se tenga un sesgo de selección y no necesariamente los resultados obtenidos en este trabajo sean extrapolables a otras poblaciones. No obstante, al comparar el comportamiento de los factores de riesgo estudiados, se encontró que estos son similares a lo reportado en la ENSA 2000 21, resultando los indicadores antropométricos y la edad los riesgos de mayor importancia para la diabetes mellitus tipo 2.

En este trabajo, se encontró una mayor sensibilidad y una menor especificidad para las pruebas utilizadas, y en los trabajos reportados por la OMS se obtiene una menor sensibilidad y una mayor especificidad; así, del total de población en riesgo de los trabajos señalados con la primera prueba, que es un cuestionario de factores de riesgo, pasa a la segunda fase del tamizaje aproximadamente el $14 \%$ de la población. En este trabajo, se encontró que del total de la población en riesgo identificada, también a través del cuestionario de factores de riesgo, pasa a la segunda prueba el $77,7 \%$ de los participantes.

En este trabajo, el VPP más alto se alcanzó con la aplicación completa del programa, lo cual es parecido a lo obtenido con solamente la medición de glucosa capilar; con lo cual se puede suponer que independientemente de la positividad de la primera prueba, la medición de la glucosa capilar indica que 16 de cada 100 sujetos son realmente diabéticos.

Según los cálculos, en México, el mayor gasto en salud destinado a la diabetes mellitus tipo 2 es para medicamentos, y para la atención de sus complicaciones, y el menor recurso se destina al diagnóstico y la consulta médica 5 . Pese a que los resultados en este trabajo pueden no ser representativos a nivel nacional, ni son concluyentes, el VPP obtenido, apunta a que una alta proporción del gasto de los servicios de salud es para descartar a los falsos positivos. Por la magnitud del problema, y el gran costo económico y social que significa la diabetes mellitus tipo 2, es conveniente considerar trabajos que puedan retroalimentar el gran esfuerzo de la Secretaría de Salud Nacional y optimizar los criterios utilizados en el PADM-2, para que la población sea la mayormente beneficiada. 


\section{Resumen}

Desde la salud pública, los programas de detección para diabetes mellitus tipo 2 son un recurso para su vigilancia. La Secretaría de Salud Nacional implementó como estrategia de tamizaje el Programa de Acción Diabetes Mellitus (PADM-2), el cual se basa en dos pruebas secuenciales: Cuestionario de Factores de Riesgo y medición de glucemia capilar. En este trabajo, se exploró la capacidad del PADM-2 como estrategia de tamizaje. El estudio se efectuó en el año 2005, participaron 1.562 individuos que asistieron a seis unidades del primer nivel de atención de la Secretaría de Salud del Gobierno del Distrito. Se consideró como el estándar de oro a la glucosa sérica en ayuno. Al valorar las dos pruebas en serie, la sensibilidad fue de 98\%, la especificidad de 58,7\%, y el valor predictivo positivo de 16,6\%. El valor predictivo positivo apunta a que una alta proporción del gasto se destina a detectar falsos positivos, lo cual hace necesario replantearse los criterios utilizados en el PADM-2, de tal manera que se obtenga un mayor beneficio económico y social.

Diabetes Mellitus; Planes y Programas de Salud; Factores de Riesgo

\section{Colaboradores}

M. G. Alvear-Galindo participó en el diseño y trabajo de campo del estudio, el análisis de la información y en la elaboración del documento. A. C. Laurell participó en el diseño del estudio y la redacción final del documento.

\section{Agradecimientos}

Al Dr. Juan José García García, profesor del Departamento de Salud Pública de la Facultad de Medicina de la Universidad Nacional Autónoma de México por su colaboración en el cálculo, la interpretación y el análisis de los valores predictivos y su comportamiento con diferentes prevalencias.

\section{Referencias}

1. International Diabetes Federation. Diabetes: atlas 2000. htpp://www.eatlas.idf.org (accedido el 07/ $\mathrm{Jul} / 2006)$.

2. Secretaría de Salud. Programa sectorial de salud 2007-2012. http://portal.salud.gob.mx/conteni dos/programa_nacional/programa_07.html (accedido el 12/Sep/2008).

3. Ramlo-Halsed BA, Edelman SV. The natural history of type 2 diabetes: practical points to consider in developing prevention and treatment strategies. Clin Diabetes 2000; 2:80-4.

4. Klein S, Sheard NF, Pi-Sunyer X, Daly A, WylieRosett J, Kulkarni K, et al. Weight management through lifestyle modification for the prevention and management of type 2 diabetes: rationale and strategies. A statement of the American Diabetes Association, the North American Association for the Study of Obesity, and the American Society for Clinical Nutrition. Am J Clin Nutr 2004; 80:257-63.
5. Arredondo A, Barceló A. The economic burden of out-of-pocket medical expenditures for patients seeking diabetes care in México. Diabetologia 2007; 50:2408-9.

6. American Diabetes Association. Standards of medical care in diabetes 2006. Diabetes Care 2006; 29 Suppl 1:S4-42.

7. American Diabetes Association. Could you have diabetes and not know it? http://www.diabetes. org/risk-test/text-version.jsp (accedido el 05/ $\mathrm{Jul} / 2006)$

8. Herman WH, Smith PJ, Thompson TJ, Engelgau $\mathrm{M}$, Aubert RE. A new and simple questionnaire to identify people at increased risk for undiagnosed diabetes. Diabetes Care 1995; 18:382-7.

9. Park PJ, Griffin SJ, Sargeant L, Wareham NJ. The performance of a risk score in predicting undiagnosed hyperglycemia. Diabetes Care 2002; 25:984-8. 
10. Glümer C, Carstensen B, Sandbaek A, Lauritzen T, Jørgensen T, Borch-Johnsen K. A Danish diabetes risk score for targeted screening: the Inter99 study. Diabetes Care 2004; 27:727-33.

11. Rolka RB, Narayan KM, Thompson TJ, Goldman D, Lindenmayer J, Alich K, et al. Performance of recommended screening tests for undiagnosed diabetes and dysglycemia. Diabetes Care 2001; 24:1899-903.

12. Dallo FJ, Weller SC. Effectiveness of diabetes mellitus screening recommendations. Proc Natl Acad Sci U S A 2003; 100:10574-9.

13. Johnson SL, Tabaei BP, Herman WH. The efficacy and cost of alternative strategies for systematic screening for type 2 diabetes in the U.S. population 45-74 years of age. Diabetes Care 2005; 28:307-11.

14. American Diabetes Association. Executive summary: standards of medical care in diabetes 2008. Diabetes Care 2008; 31 Suppl 1:S5-11.

15. Colagiuri S, Hussain Z, Zimmet P, Cameron A, Shaw J; AusDiab. Screening for type 2 diabetes and impaired glucose metabolism: the Australian experience. Diabetes Care 2004; 27:367-71.

16. Saaristo T, Peltonen M, Keinänen-Kiukaanniemi S, Vanhala M, Saltevo J, Niskanen L, et al. National type 2 diabetes prevention programme in Finland: FIN-D2D. Int J Circumpolar Health 2007; 66:101-12.

17. Nucci LB, Toscazo CM, Maia AL, Fonseca CD, Britto MM, Duncan BB, et al. A nationwide population screening program for diabetes in Brazil. Rev Panam Salud Pública 2004; 16:320-7.

18. Secretaría de Salud. Programa Nacional de Salud 2001-2006. http://www.salud.gob.mx/unidades/ evaluacion/metas_pns/metas_pns.htm (accedido el 27/Ene/2008).

19. Secretaría de Salud. Programa de acción: diabetes mellitus. México DF: Secretaría de Salud; 2001.

20. Departamento de Asistencia Técnica y Análisis del Laboratorio Auxiliar, Escuela Nacional de Ciencias Biológicas, Instituto Politécnico Nacional. Reporte $n^{\circ}$. A-237-2005. México DF: Instituto Politécnico Nacional; 2005.

21. Olaiz G, Rojas R, Barquera S, Shamah T, Aguilar C, Cravioto P, et al. Encuesta Nacional de Salud 2000. Tomo 2. La salud de los adultos. México DF: Instituto Nacional de Salud Pública; 2003.
22. Herman WH, Brandle M, Zhang P, Williamson DF, Ratner RE, Lachin J. Cost associated with the primary prevention of type 2 diabetes mellitus in the Diabetes Prevention Program. Diabetes Care 2003; 26:36-47.

23. Velázquez-Monroy O, Lara-Esqueda A, MartínezMarroquín MY, Márquez-Flores F. La detección integrada como un instrumento para vincular la prevención primaria, el tratamiento temprano, y la vigilancia epidemiológica en diabetes e hipertensión arterial. Rev Endocrinol Nutr 2000; 8:129-35.

24. Vázquez-Martínez JL, Gómez-Dantés H, Fernández-Cantón S. Diabetes mellitus en población adulta del IMSS. Resultados de la Encuesta Nacional de Salud 2000. Rev Méd IMSS 2006; 44:13-26.

25. Bautista-Martínez S, Aguilar-Salinas CA, Lerman I, Velasco ML, Castellanos R, Zenteno E, et al. Diabetes knowledge and its determinants in a Mexican population. Diabetes Educ 1999; 25:374-81.

26. Peterson K, Silverstein J, Kaufman F, Warren-Boulton E. Management of type 2 diabetes in youth: an update. Am Fam Physician 2007; 76:658-64.

27. Avila-Curiel A, Shamah-Levy T, Chávez-Villasana A, Galindo-Gómez C. Encuesta urbana de alimentación y nutrición en la Zona Metropolitana de la Ciudad de México (ENURBAL2002). México DF: Instituto Nacional de Ciencias de la Nutrición Salvador Zubirán; 2003.

28. Simons RK, Harding A-H, Wareham NJ, Griffin SJ. Do simple questions about diet and physical activity help to identify those at risk of type 2 diabetes? Diabet Med 2007; 24:830-5.

29. Rahman M, Simmons RK, Harding AH, Wareham NJ, Griffin SJ. A simple risk score identifies individuals at high risk of developing type 2 diabetes: a prospective cohort study. Fam Pract 2008; 25:191-6.

30. Ávila-Curiel A, Shaman-Lavy T, Galindo-Gómez C, Cuevas-Nasu L, Moreno-Macías H, Chavez-Villasana A. La diabetes mellitus en estratos socioeconómicos bajos de la ciudad de México: un problema relevante. Rev Invest Clín 2007; 59:246-55.

31. Dode MASO, Santos IS. Validade do auto-relato de diabetes mellitus gestacional no pós-parto imediato. Cad Saúde Pública 2009; 25:251-8.

32. World Health Organization. Screening for type 2 diabetes. Report of a World Health Organization and International Diabetes Federation meeting. Geneva: World Health Organization; 2003.

Recibido el 20/Oct/2008

Versión final presentada el 30/Sep/2009

Aprobado el 10/Nov/2009 